\title{
Germanica
}

\section{Lucebert : «as alles» [cendre tout]}

\section{Anja de Feijter}

Traducteur : Spiros Macris

\section{OpenEdition \\ Journals}

Édition électronique

URL : http://journals.openedition.org/germanica/1946

DOI : 10.4000/germanica. 1946

ISSN : 2107-0784

\section{Éditeur}

Université de Lille

\section{Édition imprimée}

Date de publication : 31 décembre 1996

Pagination : 117-141

ISSN : 0984-2632

\section{Référence électronique}

Anja de Feijter, «Lucebert : «as alles» [cendre tout]», Germanica [En ligne], 19 | 1996, mis en ligne le 04 juin 2013, consulté le 06 octobre 2020. URL : http://journals.openedition.org/germanica/1946 ; DOI : https://doi.org/10.4000/germanica.1946

Ce document a été généré automatiquement le 6 octobre 2020.

(c) Tous droits réservés 


\title{
Lucebert : «as alles» [cendre tout]
}

\author{
Anja de Feijter \\ Traduction : Spiros Macris
}

\section{NOTE DE L'AUTEUR}

Les citations de la Bible sont faites selon l'édition de Louis Segond, Paris, 1919. (NdT)

1 La poésie de Lucebert peut se définir comme une difficile profération à rencontre de la parole de Dieu. Le poète s'oppose à la parole divine. Ceci s'accompagne parfois d'agressivité, mais plus souvent de désespoir et le vers souvent cité : «ik bouw nauwgezet en wanhopig» [je construis de manière exacte et désespérée] est bien à propos parce que " construire » signifie chez Lucebert parler. Le combat qui est engagé dans cette œuvre contre la parole de Dieu, est placé sous le signe de l'émancipation de la parole de l'homme.

2 La poésie de Lucebert est pénétrée de l'opposition contre la parole divine en tant que force créatrice. La parole divine est la force qui a donné forme à toute chose et dont les traces sont partout perceptibles ; c'est surtout la force qui forma le langage. La parole divine est profondément gravée dans le premier et principal moyen d'expression dont l'homme dispose. L'idée que Dieu a donné forme à la création par la parole est familière à tout lecteur des premiers versets de la Genèse. L'accent mis sur le langage comme le moyen de création par excellence, et l'idée que la parole divine est comme une matrice ou un étalon de la création en général et du langage en particulier, est un trait caractéristique de la Kabbale, tradition mystique qui s'est développée à partir de la religion juive et dont l'histoire remonte au début de l'ère chrétienne. Dans la Kabbale, le Dieu créateur est compris comme celui qui déploie la force de la parole, fait valoir celle-ci comme étant créatrice. Dieu est doué d'une voix, il est celui qui parle et, très précisément, celui qui nomme.

3 En opposition au langage formé et donné par Dieu, Lucebert conçoit un programme de construction d'une langue nouvelle. Selon la seconde partie du titre de son recueil considéré comme son véritable début, apocrief/ de analphabetische naam [apocryphe / le 
nom analphabétique], cette nouvelle langue devra être analphabétique. Avant Lucebert, le néerlandais ne disposait que du substantif «analfabeet» [analphabète] et de l'adjectif et adverbe «alfabetisch» [alphabétique]; il n'est certainement pas sans importance que le mot «analphabetisch» soit un néologisme. Cette curieuse manière de désigner la langue à construire comme étant « analphabétique » constitue la clé de l'interprétation de la poésie de Lucebert comme s'opposant à la parole de Dieu. Avant que Dieu, le créateur-proférateur qui nomme ou dénomme, ne créa « tout » et ne donna à chaque chose un nom, il se donna tout d'abord à lui-même un nom. La langue donnée par Dieu est réductible à cette première et unique racine. Parce que le nom de Dieu est compris dans la Kabbale comme étant « le nom alphabétique »- une définition qui repose sur le lien que l'on peut faire entre le Tétragrammaton et l'alphabet - le combat pour une langue nouvelle avec des mots ou des noms nouveaux prend la forme d'un combat pour « une langue analphabétique ».

4 La langue nouvelle que Lucebert a concrétisée dans les termes «le nom analphabétique » trouve son origine dans la Kabbale et, plus exactement, doit son existence à une réaction contre une conception propre à la Kabbale. Afin d'éclairer cette proposition, j'aborderai en première instance un petit groupe de poèmes christiques puis je m'arrêterai aux principaux poèmes dans lesquels Dieu apparaît selon la Kabbale, comme celui qui nomme. En m'appuyant sur des poèmes anciens et récents consacrés au Christ, j'espère montrer les rapports existant entre le début et la fin de l'œuvre de Lucebert. Ces rapports peuvent servir d'indicateur puissant en faveur d'une impulsion constante dans l'œuvre du poète, énergie qui est puisée selon moi dans la conviction que la parole poétique doit prendre position contre la parole de Dieu.

5 Lucebert est le pseudonyme de Lubertus Jacobus Swaanswijk (1924-1994). La meilleure définition que l'on puisse donner de l'homme serait celle de poète-peintre, bien que cet artiste au double talent se soit également exprimé par la photographie et la céramique. Nous nous attacherons ici au poète.

6 L'activité du poète présente des variations remarquables. Pour ce qui concerne sa production, rien ne faisait obstacle à un début en 1950, mais le poète eut du mal à trouver un éditeur et sa première publication n'aura lieu que l'année suivante. La parution de trois recueils dans les années 1951 et 1952 souligne encore le retard.

7 Le recueil apocrief / de analphabetische naam, considéré comme son véritable premier recueil parce qu'il fut le premier proposé à un éditeur, paraît vers le milieu de l'année 1952. À partir de 1953, Lucebert publie avec une grande régularité un nouveau recueil tous les deux ans. Dans le courant des années soixante le plasticien prend le dessus et le peintre prime le poète. De fait, au début des années soixante-dix, paraît une édition monumentale de l'Euvre poétique. Mais depuis le poète a surpris par de nouvelles publications. À partir du début des années quatre-vingt, l'œuvre poétique s'accroît de cinq nouveaux recueils parus entre 1981 et 1994.

8 Il est évident qu'une parole qui s'oppose à la parole divine rencontre presque inévitablement le Christ. Cette rencontre est notamment suscitée par le début de l'Évangile de Jean, où le Christ est décrit comme étant la Parole faite chair (Jean $1: 14$ ).

Dans le récent recueil van de roerloze woelgeest [de l'agitateur immobile] (1993) se trouve le poème «glorie » [gloire] qui aborde la mort du Christ. La phrase centrale est «ij is het wel gewend te sterven» [il y est bien habitué, à mourir]. Une série d'impératifs encadrés par «druk hem aan het hart» [pressez-le sur le cœur] donne une première image du Christ et prépare à la séparation entre croyants et non-croyants. Au milieu du poème, à partir 
de la phrase consternante «hij is het wel gewend te sterven», est évoqué l'opposition entre guerre et paix. La fin est composée d'une série de prédicats enchaînés sans conjonctions, qui ont tous une valeur négative et dont le point culminant est constitué par la mort sans signe d'ordre.

Le Christ est dépeint de façon remarquablement martiale. Dans le deuxième vers commence une série de termes liés sur le plan sémantique qui ont en commun la signification «militaire ». Cette isotopie militaire qui détermine la tonalité du poème tout entier se clôt sur le terme inquiétant «keurbende» [troupe d'élite]. Du Christ, à qui est attribué cette «keurbende», il est dit «hij is het wel gewend te sterven»; une mort solitaire sans aucun signe distinctif est réservé à ceux qui n'appartiennent pas à cette élite et qui sont dits «afgekeurd» [refusés].

11 Un deuxième poème dans le recueil van de roerloze woelgeest prend pour thème la mort du Christ, il s'agit de «oh dolorosa». Le titre fait allusion à la via dolorosa, le chemin qu'a emprunté le Christ pour aller du tribunal de Ponce Pilate au Golgotha :

oh dolorosa

oh dolorosa wat was leek zo goed

oh dolorosa wat was was niet goed

het lijf zwijgt voorgoed en schijnt zwart

rode ruzie stijgt op uit het zwarte hart

krompraten mag nu dat mag de hangman

die hangt bungelt aan de gekromde rug

een oude brug naar het lege plein

waar wordt gezwegen zo goed zo goed

dat men de vloek hoort van de zwijgende maan

over de nagelnieuwe lijken op het plein

die bij het ochtendgloren verwijderd

in de nacht weer worden aangedragen

oh dolorosa het zuinige huilen

nadert de gulle lach onraad onwaar

(de estheet als bedelmonnik op een bruin

duits schilderij of misbaksel bij

een frans banket) waar naast onwaar

een dode dove taal onleesbaar

behalve voor wie zieh krap zet en hard

hardnekkig zegt dit versta ik zeer goed

dit hoor ik duidelijk ja overduidelijk

dit ruisen in mijn kromgetrokken oren

is een dwaalleer vol aangepaste dogma's

mijn eigenrede waar en onwaar in geloken oren

de houten rozen waarvan men vuurtjes stookt

op het plein op het soms lege soms overvolle plein

vuren tussen de bloedende honden de brug

dondert in duigen de houten rozen vallen

als bloedende tongen uit brandende monden

oh dolorosa nu zwijgen is lijfstraf

oh dolorosa

oh dolorosa ce qui fut parut si bien

oh dolorosa ce qui fut ne fut pas bien

le corps se tait pour toujours et paraît noir

rouge querelle monte du cœur noir

parler tordu est autorisé maintenant

autorisé l'homme pendu

qui pend pendille au dos tordu 


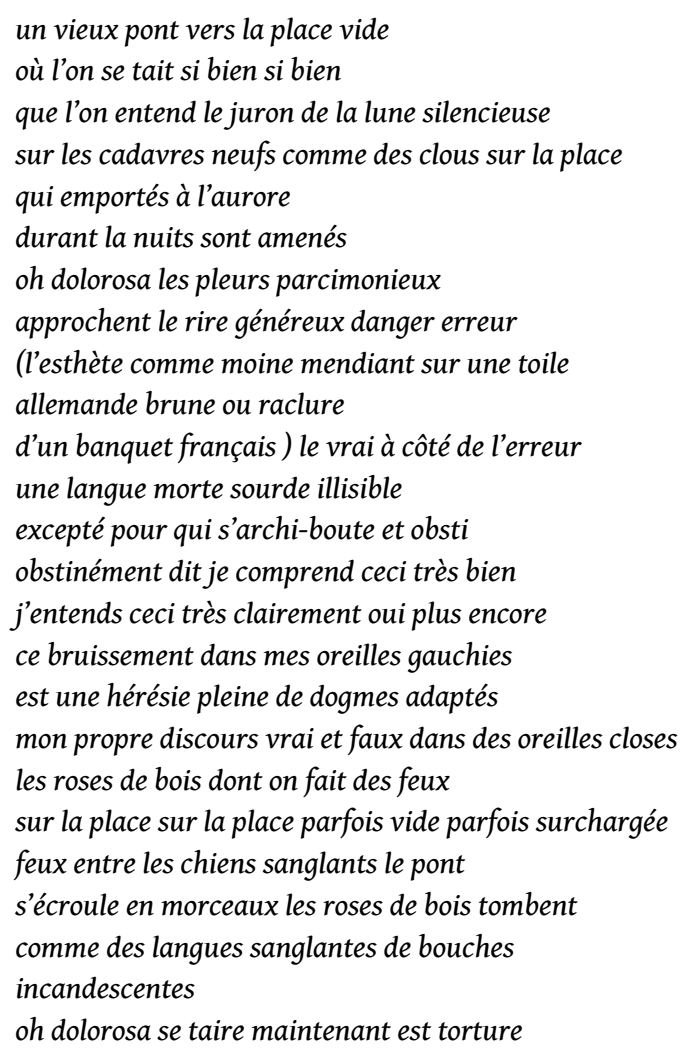

Le terme inquiétant est ici «nagelnieuw» (v. 10) [neuf comme un ongle ou un clou]. Il est peut-être bon de noter qu'il ne s'agit pas d'un néologisme, mais d'un mot figurant dans le dictionnaire et signifiant « tout à fait neuf ». Par la position que le mot occupe dans le poème, il devient cependant presque un néologisme, portant le sens de «clouer (de nouveau) le Christ sur la croix ». Il est question de «nagelnieuwe lijken» [des corps neufs] qui sont rassemblés sur la place, laquelle sera ensuite décrite comme «het soms lege soms overvolle plein» (v. 26) [la place parfois vide parfois surchargée]. L'idée est ainsi évoquée d'une répétition inévitable de la crucifixion du Christ, accumulant une telle quantité de cadavres qu'il faut leur réserver toute une place.

13 J'attire l'attention sur une particularité de ce poème qui caractérise l'ensemble de l'œuvre de Lucebert. Le mot «dolorosa» est décomposé au fil du texte. Cet éclatement procure au poète le terme «rozen» [roses]. Que Lucebert ait en effet voulu mettre en évidence «rozen» dans le mot "dolorosa» apparaît clairement dans le syntagme où figure «rozen». Dans le groupe «houten rozen» [roses en bois], répété aux vers 25 et 28, le déterminant «houten» [en bois] renvoie à la via dolorosa qui conduit à la croix.

composant les mots, Lucebert ne recule donc pas - évidemment pas, dirais-je même - devant la distinction entre les langues. Il apparaîtra en effet qu'il ne craint pas les différences entre les langues. Cet aspect semble également présent dans «oh dolorosa» ou les mots "duits schilderij of... frans banket» [une toile allemande ou un banquet français] figurent, groupe dans lequel on pourrait lire l'étreinte de différentes langues ou cultures. C'est comme si la distinction entre les langues, et donc la distinction entre les pays et les peuples, doit surtout être considérée comme une frontière à franchir.

Le lien entre la figure du Christ et le langage n'apparait pas nettement dans " glorie ", alors que c'est bien le cas de «oh dolorosa». Dans les mots «het lijf zwijgt voorgoed» (v. 3) [le corps se tait pour toujours], on reconnaît la conception johannique de la parole 
faite chair. La mort du Christ est présentée comme une condamnation de la parole au silence. Sur la place, où ne règne pas simplement le silence mais «waar wordt gezwegen» (v. 8) [où l'on se tait], on entend une non-parole paradoxale, se taire est rendu par une manière de parole «de vloek... van de zwijgende maan» [le juron... de la lune se taisant]. Dans la deuxième strophe il est question de «een dode dove taal» (v. 18) [une langue morte et sourde]. Bien qu'étant morte et sourde, cette langue est encore déchiffrable. Elle est «onleesbaar / behalve voor wie zieh skrap zet» [illisible / sauf pour celui qui s'arc-boute]. L'étrange remplacement de «schrap» par «krap» [étroit] dans l'expression «zieh schrap zetten» [s'arc-bouter] doit probablement être mis en rapport avec l'impossibilité d'utiliser dans le contexte un terme qui pourrait renvoyer au verbe «schrappen» [rayer, racler] et ainsi à «een woord schrappen» [rayer un mot]. Le Christ mort est si audible ou présent qu'il ne saurait être question de rayer un mot. Le mot effectivement utilisé, «krap», est relié, par équivalence phonologique, à tous les mots construits avec «krom» [courbé, tordu] : «krompraten» [parler de manière tordue] (v. 5), «gekromde» [courbé] (v. 6), «kromgetrokken» [gauchi] (v. 22). De la sorte, il ne s'établit pas seulement un rapport entre «krompraten» [parler de manière tordue] et le dos tordu du Christ sur la croix, mais également entre le Christ dont le dos est tordu et quiconque s'oppose à l'illisibilité de cette langue morte. Le qualificatif « illisible » est suivi par une double description. La «dode taal» est décrite et comme «een dwaalleer» (v. 23) [hérésie] et comme «mijn eigenrede» (v. 24) [mon proprediscours / propreraison]. La langue dont il est ici question est refusée en tant que doctrine fausse, mais en même temps - c'est là un signe de désespoir - reconnue par le poète comme son propre moyen d'expression. Le fait d'être condamné à la langue donnée par Dieu rend la parole d'autant plus nécessaire. J'interprète le dernier vers, «oh dolorosa nu zwijgen is lijfstraf» [oh dolorosa se taire maintenant est supplice] comme le plaidoyer passionné pour une parole s'opposant à la parole de Dieu.

16 Ma conclusion est qu'il y a place pour différentes figures du Christ dans van de roerloze woelgeest. Le poète met aussi bien en scène un Christ martial, qu'un Christ sur le chemin de croix. Le Christ des douleurs est la parole faite chair, dont le supplice et la mort sont présentés comme une condamnation au silence et dont la vie est en rapport direct avec la langue dont le poète dispose.

Par l'association entre la mort et la surdité, «oh dolorosa» est étroitement lié à un des poèmes les plus célèbres et les plus importants d'apocrief/ de analphabetische naam, premier recueil de Lucebert. Je pense à l'« introduction » à «lente-suite voor lilith» [suite printanière pour lilith]. Il s'agit de l'introduction à un ensemble de trois textes dans lesquels le poète fait de Lilith sa muse. On ne saurait accorder assez d'attention au fait que nous avons affaire ici à la même "Umwertung aller Werte» nietzschéenne que dans de analphabetische naam. Lilith est une des créations les plus spectaculaires de la Kabbale. Elle est la figure sur les fragiles épaules de laquelle repose le poids de tous les péchés, notamment ceux touchant le domaine de la sexualité. Lucebert libère cette figure de la malédiction qui pèse sur elle et fait de cette furie la muse de sa poésie, nymphe enjôleuse qui mérite une ode.

Le poème «introduction" est déterminé par l'opposition entre "lumière " et « obscurité » et les différences entre des poètes aux options divergentes occupent une place centrale. Les poètes qui ne se manifestent pas, ou pas assez, sont morts et sourds. Ils sont ironiquement décrits comme des orties blanches [dovenetel; dove = sourd]. Le poème est étroitement lié au contexte littéraire du moment, de sorte que l'on reconnaît 
dans les quelques poètes qui s'opposent aux poètes-orties blanches et pour lesquels Lucebert joue le rôle de porte-parole, des représentants du Mouvement de (19)50. Le point essentiel de la poétique de cette nouvelle génération de poètes est de maintenir ouvertes les frontières entre la lumière et l'obscurité ou le bien et le mal. Un des sens que renferment ces vers : «alleen wat dichters brengen het te weeg / uit poelen worden lelies opgedregd» [seul quelques poètes y atteignent / dans les mares on drague des lis] est que la lumière peut être puisée dans l'obscurité et qu'une séparation a priori entre le bien et le mal n'est pas défendable.

19 «Introduction » constitue un premier pas en direction de l'œuvre la plus ancienne de Lucebert. Celle-ci comprend pour moi non seulement apocrief/de analphabetische naam, mais également les poèmes de 1949-51 qui précèdent le premier recueil et n'ont pas été repris en volume.

Le Christ apparaît également de diverses manières dans ces poèmes. Dans le long poème " exodus ", c'est le dernier des personnages principaux. La représentation du Christ en tant que dernier a été inspirée à Lucebert par Hölderlin qui, à peu près un siècle et demi plus tôt, a donné une image tout à fait particulière du Christ. Hölderlin a tenté de rendre pour ainsi dire le Christ moins seul en le plaçant sur le même plan que des autres fils de Dieu et en le décrivant comme le dernier rejeton d'une vaste famille divine composée des grandes figures de l'antiquité et du christianisme.

21 «Exodus» ne se réfère pas seulement par la représentation du Christ comme dernier à Hölderlin, mais également par un ensemble de six vers qui se présentent comme une ode adressée par Lucebert au poète allemand ; prédécesseur pour lequel il a manifesté son admiration ailleurs dans son œuvre. Arrêtons-nous un instant sur ce passage. Un des motifs conduisant à lire dans le verbe «bouwen» [construire] le sens de "parler", pourrait être emprunté à Hölderlin. Il a uni le permanent et le poétique dans le vers «Was bleibet aber, stiften die Dichter». Il met également en rapport le verbe «stiften» avec Dionysos, un des dieux qu'il place aux côtés du Christ. Lucebert rend le «stiften» holderlinien concret et présente la création des poètes comme la réalisation d'immeubles [gebouwen] durables :

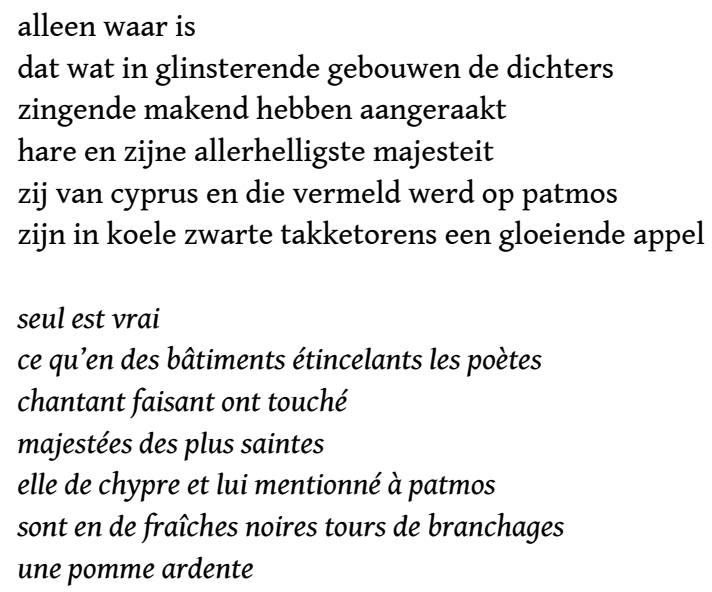

Ces vers de Lucebert se laissent interpréter comme une ode dédiée à Hölderlin principalement par ce qu'il crée un couple syncrétique semblable au couple formé par le poète allemand avec le Christ et Dionysos. Avec les «twee heilige majesteiten» [deux majestés saintes], Lucebert évoque, par le biais des îles - Chypre et Patmos - auxquelles elles sont associées, deux figures majeures de l'antiquité et du christianisme: Aphrodite et Jean. Que ce soit justement Jean de Patmos que Lucebert ait placé dans son 
ode à Hölderlin n'est pas sans importance. Il est vrai qu'Aphrodite de Chypre et Jean de Patmos figurent tous les deux dans l'œuvre de Hölderlin, mais c'est surtout Jean qui, grâce à l'hymne « Patmos », apparaît comme personnage central dans l'œuvre du poète allemand.

Le lien entre la langue et la question du Christ est évident dans un poème de apocrief/ de analphabetische naam où Lucebert se sert pour ainsi dire de Jean pour dépeindre le Christ. Dans le poème ayant pour titre «het vlees is woord geworden» [la chair s'est faite parole] les termes de l'évangile de Jean sont inversés. Les dévots représentés dans le poème sont des poètes et/ou des lecteurs qui se penchent sur le "droomkadaster» [cadastre de rêve] du lexique ou du dictionnaire. Ce dont il s'agit, c'est que la parole ou le Christ est la victime de leurs recherches.

Pour la construction d'une langue nouvelle on peut procéder de différentes manières. Lucebert ne veut ni ne peut partir de rien. Il est question bien plus de pénétrer dans les « oubliettes» ou la « cage » du langage pour l'attaquer de l'intérieur. Le premier vers de "de kooien van de poëzie» [les cages de la poésie] donne le ton de l'imbrication remarquable du poétique et de l'orde animal qui englobe le poème dans son ensemble. Des poètes sont associés aux animaux de Miro [«het gedierte van Miro»], et plus particulièrement aux puces, hannetons et grenouilles. Les puces, hannetons et grenouilles ont ceci en commun qu'ils changent d'apparence au cours de leur vie; le terme principal de cette isotopie animalière est sans doute le dernier: "maden» [larves]. Dans un article remarquable consacré aux rapports entre Miro et Lucebert, Lieve Scheer cite dès la deuxième page toutes sortes d'animaux, entre autres des têtards, lombrics et autres vers de terre. L'auteur parle d'« étrange vermine boschienne [...] pullulant partout chez Miro avec une vitalité inextinguible». Cette vitalité se retrouve également dans «het vlees is woord geworden», énergie créatrice irrépressible, cause de la métamorphose des mots anciens en mots nouveaux. Des poètes entrent dans la cage de la langue avec la volonté de créer. Plus largement, on couve dans les cages de la littérature et de la peinture aussi bien des mots nouveaux que des images nouvelles. Les œuvres des nouveaux artistes, productions de leur "langue » et de leurs " yeux », mots et images nouveaux, sont des " enfants » comme l'enfant de la crèche de Noël.

Le langage est attaqué de l'intérieur. Dans «gevoelig Vatikaan» [Vatican sensible], les poètes exercent la puissance de leur voix pour ensuite laisser rouler leurs cris sur le «ijshouten woud» [forêt de bois glace]. Parce que «Vatikaan» et «woud» sont opposés sur le mode sensible insensible, je suppose que le langage est également présenté comme une forêt glacée, une construction grandiose de nombreux arbres ou d'un seul se ramifiant puissamment. Le symbolisme de la Kabbale offre des points de repères pour cette image. Le «bois » n'est pas seulement présent dans «woud», mais également dans «het geraamte van de kerststal» [l'armature de la crèche de Noël]. Les poètes en coassant précipitent leurs cris, comparés à des " grenades », sur le «ijshouten woud» du langage. Leur activité a pour résultat de fissurer quelque peu le bois glacé, séparant des fragments, du «cristal». Il ne s'agit cependant pas simplement d'éclats ou d'esquilles de glace qui pourraient être comparés au cristal, mais à des «maden van kristal» [larves de cristal]. Ces larves de cristal se métamorphosent à leur tour en «tranen van de dood» [larmes de la mort]. Les poètes réduisent la langue ancienne en poussière en en montrant la nature véritable ou en indiquant un sens nouveau. Que la réflexion des poètes sur la langue est 
à la fois destructive et constructive, voilà ce qu'indique surtout à mon avis la comparaison avec l'orage. Le souffle poétique se pose comme une cloche sur les mots tout comme un ciel d'orage recouvre les bâtiments des banques. Les «bankgebouwen», nouvelle métaphore des mots, se mettent à «ruisen van inflatiegerucht» [bruire de rumeurs d'inflation]. Le fruit de la destruction est l'éclair ou la fulguration d'une idée, de la révélation d'un sens nouveau. Le sens révélé ici est que le Christ ne peut même pas être compris comme celui à qui n'est réservé qu'une courte vie, une vie comme un souffle. Il est présenté comme étant destiné à la mort, de telle manière qu'enfant dans la Crèche il portait déjà les signes de la crucifixion.

Un poète d'inspiration mystique qui recherche Dieu en l'étudiant ne pourra manquer le vers inaugural de l'évangile de Jean: «Au commencement était la Parole, et la Parole était avec Dieu, et la Parole était Dieu. " (Jean 1:1). Les premiers mots de Jean forment l'hypogramme du début et de la fin de l'un des poèmes des années 1949-51 qui n'ont pas été repris en volume. Je l'aborde ici pour faire une transition vers le poème où Dieu se manifeste comme «noemer» [celui qui nomme].

La première moitié de «meditatie op een mond vol builenbal» [méditation sur une bouche pleine de boules] traite de la création de l'homme et de la femme. Cette création se déroule de manière extrêmement douloureuse et les touche surtout dans leur voix. Il est dit de l'homme qu'il «hurle comme un chien » et de la femme que sa voix n'est qu'un "étroit lobule dansant». Dans la deuxième partie du poème est donnée une image effrayante de la toute puissance de la voix de Dieu à l'ombre de laquelle se trouve l'impuissance de la voix humaine :

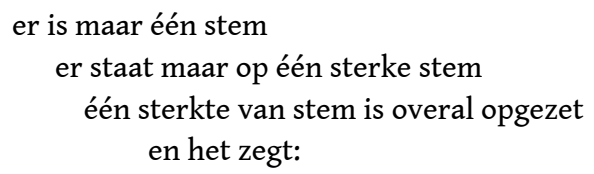

«TETTEN» recèle une référence remarquable à la Kabbale. La création par la voix de Dieu déforme quelque chose dans le visage de l'enfant. Pour expliquer cette déformation il me faut plus particulièrement faire appel au Zohar, l'œuvre principale de la Kabbale. Lucebert renvoie explicitement à cette œuvre par les mots «zie zohar» [voir zohar] dans une note insérée dans «het proefondervindelijk gedieht» [le poème expérimental], autre texte célèbre de la période 1949-51.

Le Zohar dont le nom complet est Sefer Hazohar [Le livre des splendeurs] est un ouvrage volumineux d'à peu près 2500 pages en petits caractères. Le livre a été écrit en terre castillane au cours du dernier quart du XIII ${ }^{e}$ siècle par le kabbaliste espagnol Mozes de 
Leon. L'auteur qui rédigea une série d'œuvres en hébreu dont il reconnut la paternité, fit disparaître, dans le cas du Zohar son nom au profit d'une autorité historique. Il donna donc à son œuvre la plus importante la forme d'un pseudépigraphe, œuvre dont l'auteur n'est pas celui indiqué. L'autorité historique qu'il choisit à cette fin est le rabbin Simeon ben Jochai, un des principaux représentants des docteurs de la foi de la deuxième génération, les Tannaïm ou "enseignants ", actifs depuis le début de notre ère jusque vers 220. Dans le Zohar, le rabbin Simeon déambule dans une Palestine imaginaire, dialoguant avec Eleazar, son fils, ses amis et ses élèves. En accord avec cette fiction historique et pseudépigraphique, le Zohar est rédigé en araméen.

31 Il a fallu longtemps avant de découvrir derrière le personnage du rabbin Simeon du II siècle, la figure du kabbaliste Mozes de Leon vivant au XIII ${ }^{\mathrm{e}}$. C'est là un des nombreux mérites de Gershom Scholem. La langue du Zohar pose d'immenses problèmes. Une excellente anthologie en langue anglaise est disponible depuis peu. Elle rend l'œuvre réellement accessible. Je cite, en me basant sur cette édition, le récit consacré à la lettre tet. Le Zohar est basé sur un texte de la Kabbale bien plus ancien, dans lequel on raconte que Dieu réalisa la création à l'aide de l'alphabet. Cette création découle de paires de lettres et leurs combinaisons toujours nouvelles qui se créent en faisant tourner les lettres, placées en une sorte de cercle. Le Zohar reprend cette image d'un cercle de lettres et raconte ce qu'il se passe lorsque la lettre tet, la première du mot «tow» [bien] se trouve combinée avec resh, la première lettre du mot «ra» [mal]. Alors que le processus de rotation s'interrompt (Tishby 1989, p. 526-527):

When the Holy One, blessed be He, created the world, He created it with the letters of the Torah, and every letter came into His présence, until all the letters stood by the letter bet, and all those thousand letters bet, around which the [other] letters revolved, were ready to help in the création of the world. While they were revolving, the two letters tet and resh found themselves together, and tet arose and could find no rest, so the Holy One, blessed be He, rebuked her, and said : tet, tet, why do you rise? Why can you not find rest in your place? She said to Hirn: Did you not make me the first letter of « good», my first appearance in the Torah - « ... that it was good » (Genesis 1:4) ? How can I possibly sit next to the letter of evil ? He said to her : Return to your place, for you need her. Man, whom I wish to create with you, will include both of you, and will be created so, but you will be on his right side, and she will be on his left side.

On retrouve dans les premiers poèmes de Lucebert des analogies avec ce qui a poussé la lettre tet à la contestation. Lucebert exploite la possibilité de concentrer dans la lettre resh le mal en orthographiant le nom de Barabbas - comme on le sait la figure opposée au Christ - d'une façon différente de l'orthographe habituelle. Il n'écrit pas Bar-Abbas ou Barabbas mais « barrabas » et rend ainsi la racine ra ou la racine du mal visible dans le nom.

Si l'on conçoit que Lucebert répond à la conception kabbalistique du nom alphabétique par de analphabetische naam, on acceptera plus aisément l'importance de l'orthographe particulière du nom de Barrabas. Alphabet, lettres, orthographe sont à tel point porteurs de l'opposition entre les noms alphabétique et analphabétique qu'un sens créé par une orthographe déviante n'est que trop vraisemblable. Barabbas apparaît en tant que «barrabas » dans «as alles» [cendre tout], poème dans lequel Lucebert montre Dieu comme étant « celui qui donne nom »:

as alles

melasse alles

van meethand melasse alles 


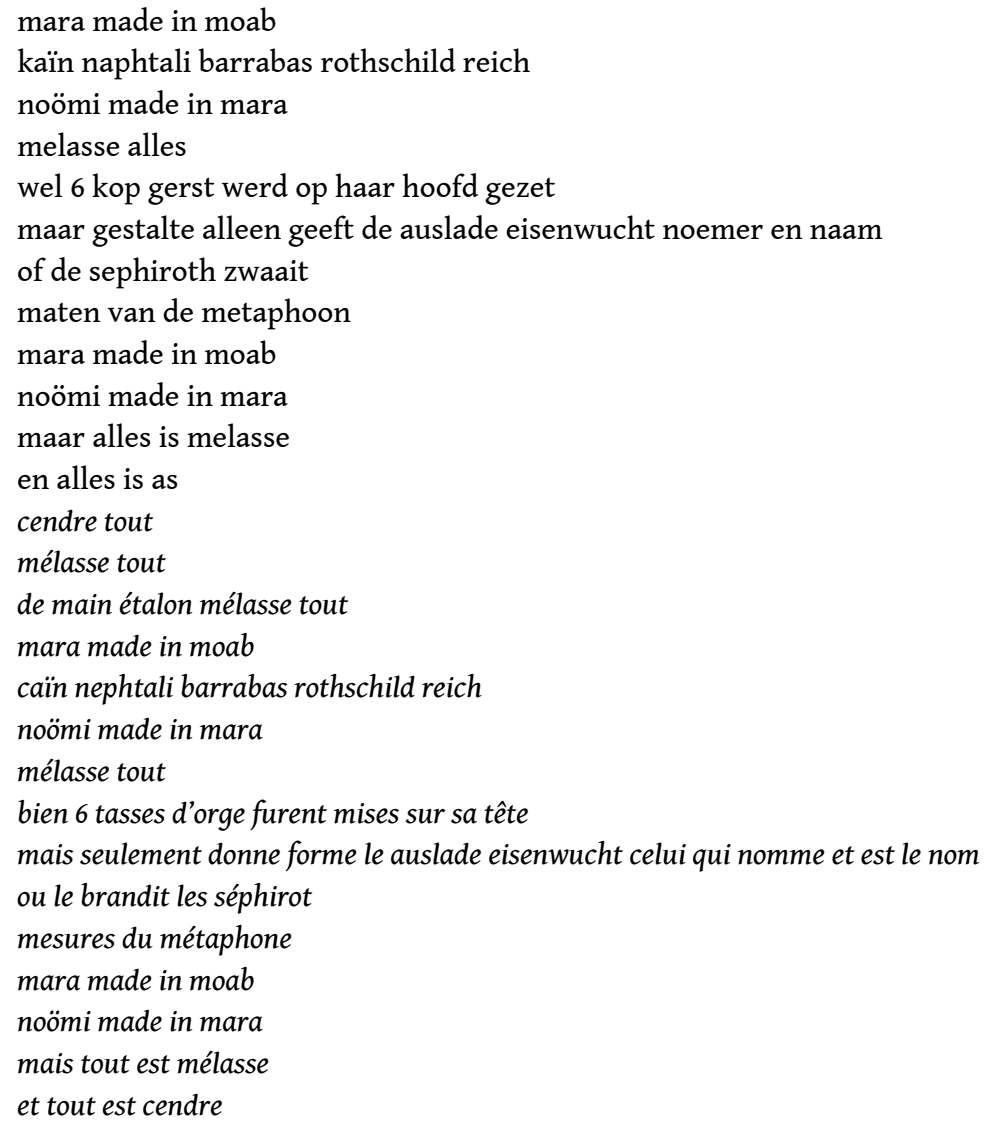

Lucebert utilise dans ce poème de ses débuts le mot étrange de "sephirot ", terminus technicus de la Kabbale par excellence. La mystique juive construit l'enseignement des séphiroth sur le texte biblique. La Kabbale est essentiellement une exégèse, une explication de la Bible en un sens mystique, afin de lever un coin du voile tendu devant le monde caché de Dieu.

Comme le livre de Ruth, le chapitre 40 du livre d'Esaïe est important pour «as alles». Dans le chapitre 40 d'Esaïe, que la Kabbale soumet sans cesse à exégèse, les deux actions de peser et de mesurer sont attribuées à Dieu. Dieu est plus particulièrement présenté comme le créateur qui mesure de sa main la création : "Qui a mesuré les eaux dans le creux de sa main, Pris les dimensions des cieux avec la paume [...] ?» (Esaïe $40: 12$ ). Dieu ne se profile pas seulement derrière le «meethand» [main étalon], mais surtout derrière «noemer» [celui qui nomme]. Le «noemer» qui «gestalte geeft [...] of de séphiroth zwaait» [donne forme [...] ou brandit les séphiroth] est Dieu. Le monde caché de Dieu est désigné dans la Kabbale simplement par "le monde des séphiroth ». La quête de Dieu est la quête de Dieu dans ce monde-ci, saturé des traces de Dieu. Le postulat de la Kabbale est qu'à la base de la création du monde se trouve la révélation de Dieu. Dieu se révèle dans les séphiroth, les sphères ou régions du monde divin et crée ce monde-ci selon le modèle du monde dans lequel il s'est révélé. Dieu peut être trouvé partout dans la création et, en dernière instance, créateur et création sont identiques. Dans la phrase complexe qui commence par «maar» (v. 9) et viole les règles de grammaire, Lucebert donne une image incroyablement frappante de Dieu tel que l'on le perçoit dans la Kabbale. La construction de la phrase se délite parce que le sujet en est Dieu. Le long vers nominal perd son équilibre parce que « Dieu » se trouve au bout. 

pour la création de l'homme. C'est pourquoi on peut interpréter «gestalte geven» et «séphiroth zwaaien» comme deux références à la même activité, celle de Dieu. Peu importe laquelle des deux tournures l'exprime. L'important est que derrière cette activité, il ne peut y avoir qu'un seul sujet. Le seul sujet possible de «gestalte geven» ou de «sephirot zwaaien», est Dieu. Cette attribution exclusive du sujet trouve notamment son expression dans la forme du prédicat. Celui-ci se déploie pour moitié à droite et pour moitié à gauche du sujet expansé à l'extrême. Dieu se trouve ainsi, en tant que personnage agissant qui détermine tout, au centre de son action, celle-ci se laissant saisir en des termes divers. Dans le sujet expansé lui-même, placé entre les deux parties du prédicat, la différentiation entre Dieu et son action disparait. On peut entendre dans le fait que les mots «noemer en naam» se trouvent dans le même syntagme-sujet, le dépassement de l'opposition entre sujet et objet. C'est là un trait caractéristique de la mystique juive qui place sur un pied d'égalité créateur et création.

Le noyau du syntagme-sujet est «noemer» [celui qui nomme], seul élément où l'on reconnait une instance agissante. Cette position particulière de «noemer» indique le primat du langage et resserre d'autant plus le lien avec les autres vers nominaux, ceux traitant de Naomi - ou Noömi selon les graphies - et le vers qui apparait comme une série de noms essentiellement bibliques.

«Barrabas» est incontestablement le centre du long vers nominal de la première strophe commençant par «kaïn». Dans cette série de noms, un certain nombre d'enchaînements produisent un lien sémantique. Ce n'est pas seulement le mal unissant Caïn et Barabbas qui joue un rôle, mais également l'argent. L'aspect financier relie Rothschild et Reich, dans lequel on entend l'adjectif «riche», avec Naomi. Dans son histoire aussi les affaires d'argent jouent un rôle important. Cette équivalence sémantique révèle la dimension financière de l'épisode de Barabbas : la condamnation de Jésus est le prix payé pour sa libération. En contre-partie, l'importance de ces deux enchaînements sémantiques est relativisée par des liens d'une autre nature dont on peut déduire que le facteur sémantique englobant l'ensemble n'appartient ni au mal ni aux sphères de l'argent.

39 Le poids des rapports d'argent est relativisé par le motif de l'émancipation. Ce caractère est emprunté à l'histoire de Naomi où les frontières entre peuples et pays sont transgressées par le mariage entre Booz et Ruth. Naomi, Rothschild et Reich sont reliés par l'ordre financier, mais autant par ce motif de l'émancipation. Tout comme la famille de Naomi et de Booz, la famille de Rothschild n'a pas négligé d'utiliser sa position sociale privilégiée à des fins d'émancipation et l'on ne peut refuser au psychanalyste autrichien Reich ces mêmes objectifs. L'importance du mal est relativisée par Nephtali, un des fils de Jacob. Nephtali se trouve placé entre Caïn et Barabbas, tous deux si nettement liés par le mal, alors que l'on ne trouve guère trace du mal en lui. Il occupe donc la place la moins évidente. La clé de l'interprétation de la fonction d'intermédiaire de Barabbas et de la série se retrouve pourtant dans son nom à lui.

L'élément sémantique englobant tous les noms est l'histoire du peuple juif. Jacob n'est pas nommé, mais sa présence est implicite par le rappel de «naphtali ». Il est le père fondateur avec le surnom d'Israël auquel le peuple d'Israël doit son nom. Les tribus d'Israël doivent leur nom à sa nombreuse postérité, constituée à partir de ses douze fils dont Nephtali fait partie. La fonction d'intermédiaire de Barabbas se déploie dans sa 
plénitude sur le plan historique où sa position est à mi-chemin entre toutes les autres figures et les relie logiquement. Barabbas est un contemporain de Jésus, qui répartit les autres entre ceux qui viennent avant et ceux qui viennent après le Christ. Il est le chaînon entre les temps anciens et les temps nouveaux reliant histoire biblique du peuple juif à son histoire moderne.

Que la série de noms dans son ensemble doive être considérée comme une histoire résumée du peuple juif peut également être déduit du fait qu'une étape curieuse est franchie dans la progression du vers. Il s'agit du passage de «barrabas» à « rothschild », qui attire l'attention sur un caractère particulier du poème dans son entier.

On entend dans «as alles» cet étrange mélange des langues, cet aspect du langage du poète sur lequel nous avons déjà attiré l'attention. Le saut d'une langue à l'autre opéré par Lucebert se retrouve à plusieurs reprises dans ses premiers poèmes, mais nulle part peut-être avec une telle intensité que dans ce poème. Dans la première strophe, le vers qui commence avec « kaïn » se trouve entouré par les vers consacrés à Naomi. Alors que dans les derniers vers l'anglais fait son apparition, c'est l'allemand qui s'introduit dans le premier. Dans la deuxième strophe, c'est l'allemand encore qui se présente tout d'abord et en un point comparable à la strophe précédente. Tout comme dans le long vers nominal de la première strophe se trouvent les noms "rothschild reich", la deuxième strophe présente dans le long vers nominal les «auslade eisenwucht». Après les mots étrangers «séphiroth» et «metaphoon», l'oscillation d'une langue à l'autre s'achève par la répétition du vers consacré à Naomi.

43 Par la référence au livre de Ruth, le mouvement de langue à langue devient perceptible comme un mouvement de pays à pays. J'interprète ces oscillations d'une langue à une autre comme l'évocation du problème du pays d'appartenance indissolublement lié à l'histoire du peuple juif. Chaque nom choisi par le poète, en porte trace et représente l'histoire d'une vie qui n'a pu échapper à ce problème.

«Kaïn» est l'enfant des "premiers chassés», chassés du paradis. «Naphtali» représente une des tribus qui a pris réellement possession de la terre promise que Moïse n'a pu que voir. Le nom de Naomi est niché entre Nephtali et Barabbas. C'est la femme qui dut quitter temporairement son pays, mais a pu y revenir. A l'époque de Barabbas sa propre terre faisait partie de l'empire romain représenté par Pilate le gouverneur. On franchit le pas vers l'histoire moderne avec le nom de " rothschild ». Le nom de la banque internationale Rothschild, fondée dans la deuxième moitié du XVIII ${ }^{\mathrm{e}}$ siècle en Allemagne et étendue sur une part importante de l'Europe au début du siècle suivant, est synonyme de diaspora. Avec «reich» enfin on atteint le $\mathrm{XX}^{\mathrm{e}}$ siècle et l'époque contemporaine. Il représente les réfugiés de notre temps, fuyant le régime national-socialiste allemand. Wilhelm Reich (1897-1957) quitte Vienne au début des années 30 pour s'installer à Berlin. Après l'arrivée au pouvoir de Hitler, il fuit en 1933 vers la Scandinavie pour se rendre enfin en 1939 aux États-Unis. Il décrit ainsi dans le temps et dans l'espace un parcours qui le rapproche de beaucoup d'autres. Il faut donc entendre dans son nom à la fois l'adjectif et le substantif de sorte que l'on doit conclure que Lucebert conclut la série de noms propres par une allusion évidente au III ${ }^{e}$ Reich.

Le dernier mot sur le choix de Nephtali parmi les fils de Jacob revient au poète luimême. Dans une lettre qu'il m'adresse en avril 1993, il écrit « que Nephtali, traduit en allemand signifie mein Kampf.» Rachel considère le combat qu'elle engage contre la stérilité et contre sa sœur Léa qui avait déjà donné des enfants à Jacob comme un combat divin. L'enfant que mit au monde sa servante eut pour nom Nephtali, «mon 
combat» ou «ma lutte", selon les paroles "J'ai lutté divinement contre ma sœur » (Gen. 30:8). Lucebert ne renvoie donc pas seulement par le nom de «reich» à l'histoire la plus récente, mais également par celui de l'un des douze fils de Jacob: le nom de " naphtali » peut se traduire par le titre du livre d'Hitler Mein Kampf. L'allemand apparaît donc dans le long vers nominal de la première strophe de différentes façons : explicite et audible dans «rothschild reich», et de manière très codée voire uniquement lisible dans « naphtali ».

«As alles» suscite avec insistance la question de la conception de Dieu. Parce que l'essence de Dieu est déterminée comme étant « celui qui nomme » et que l'histoire du peuple juif est résumée par une série de noms, l'idée se fait jour que Dieu a condamné son peuple au statut de n'être qu'une enfilade de noms.

L'idée inquiétante que la création divine n'aurait rien produit d'autre que des déchets est présente tout au long du poème. Le terme répété «as» [cendre] est compris dans l'autre mot tout autant répété «melasse» [mélasse]. La mélasse est un sous-produit ou le résidu de la production sucrière. Les mots «meethand melasse alles» montrent qu'à tout ce qui vient de la main du créateur colle l'idée de déchet. La combinaison caractéristique de l'action de mesurer et de peser avec l'acte de dire et de nommer, exprimée de manière frappante dans le vers : «maten van de metaphoon» [mesures $d u$ métaphone], indique que toute création divine, quel qu'en soit le mode, possède toujours ce caractère de déchet. Quoique Dieu crée, tout est rebut, cendre et mélasse. Le fil de l'histoire est continué jusqu'à notre époque. Il est donc inévitable d'attribuer à "as», premier et dernier mot du poème, un sens particulier. Il ne s'agit pas uniquement de la " cendre des morts ", mais plus précisément de la matière qui reste des victimes juives du régime nazi, brûlées après avoir été gazées.

La portée historique du poème est grande. Elle embrasse une période qui va de la création à un passé récent. Par ce recul historique considérable la pression sur le passé récent s'avère d'autant plus grande, comme si rien n'avait changé et que l'histoire se répète inéluctablement.

L'idée que des mains du créateur ne viennent que des déchets prend sa source dans la Kabbale. Les résidus de «as alles» conduisent à aborder le symbolisme du mal dans la Kabbale.

Les tentatives faites dans la Kabbale pour éviter le plus possible les conséquences dualistes ont conduit à une réponse surprenante à l'interrogation sur la nature et l'origine de mal. La Kabbale n'a pas reculé devant la conclusion que le mal fait partie de Dieu et le Zohar développe une métaphore osée afin de désigner le mal divin.

51 On a trouvé une solution tout à fait remarquable au dilemme selon lequel le mal n'a pas de place en Dieu qui est bon. D'après la Kabbale la racine du mal réside bel et bien en Dieu. Mais dès le début du processus d'émanation Dieu s'est débarrassé du mal qui était en lui en s'en séparant comme d'un déchet. Considérer le monde caché de Dieu comme un monde où bat le pouls de Dieu ou concevoir la vie divine scellée comme un processus organique, c'est inviter à une comparaison avec le processus organique ce qui renforce la représentation du mal comme étant un déchet divin. Le mal est considéré comme un résidu, reste du processus organique qui a lieu dans le monde des séphiroths. L'écorce de l'arbre, la cosse, la coque ou la peau d'un fruit, la lie au fond d'une bouteille de bon vin, le "mauvais sang " éliminé par la saignée ou les divers déchets produits par le corps, tous les exemples de déchets dans ce monde sont invoqués pour appuyer l'idée que la vie dans le monde caché de Dieu ne se conçoit pas 
sans déchets et que tout ce qui est démoniaque plonge ses racines dans le mystère de Dieu. syntagme-sujet. J'interprète le sujet très étendu comme une allusion aux spéculations de la Kabbale sur un certain nombre de noms de Dieu, eux aussi extrêmement longs et cachés. Le verbe allemand ausladen se traduit en néerlandais aussi bien par uitladen [décharger] que par lossen [décharger, se défaire, libérer]. De ces deux verbes, c'est surtout le dernier dans le sens d'évacuer, qui possède des connotations susceptibles d'être rapprochées du symbolisme de l'élimination. Le poème invite à cette traduction parce que Booz, l'homme qui libère Naomi de ses soucis matériels et spirituels, est appelé dans la Bible «losser». Admettons enfin que Booz et le Christ, le «verlosser» [libérateur], se trouvent sur le même plan. Le cercle évoqué dans «as alles» alors se referme avec «barrabas», araignée au centre dans la toile des relations tissées entre différents personnages.

La question de la conception de Dieu prend la forme plus précise d'une question portant sur la vision du rôle de Dieu dans l'histoire du peuple juif. L'interrogation s'impose notamment à l'endroit du rôle de Dieu dans l'exemple le plus récent de persécution de l'histoire du peuple juif. Dans le poème où il utilise l'idée de la Kabbale selon laquelle Dieu se défait du mal comme d'un déchet, Lucebert fait référence à la deuxième guerre mondiale, de sorte que la question de la responsabilité de Dieu en la matière se trouve posée.

Je considère «as alles» comme l'un des poèmes les plus impénétrables de Lucebert. Peut-être est-ce la raison pour laquelle il n'a pas été repris dans un recueil. Quoi qu'il en soit, il reste un autre poème, repris dans un recueil celui-là, qui traite du même sujet et où le poète dévoile sans fard sa conception du rôle de Dieu. Il s'agit du poème «vaalt» [dépotoir, fumier], dans le recueil apocrief / de analphabetische naam. Lucebert indique dans «vaalt» que la racine du mal se trouve dans l'idée de nation. Le poète voit le mal dans la distinction entre les peuples et rend Dieu, comme étant créateur d'un peuple qui lui appartient, responsable de cette distinction et du mal du nationalisme.

$56 \mathrm{Au}$ début de «vaalt» le ton est faussement léger. On donne au Je, qui empoigne avec bonne humeur une fourche, l'ordre de nettoyer l'«excrementenplein» [place (couverte) d'excréments]. Mais ce n'est pas du fumier ordinaire qu'il ramène avec son outil. La place est remplie de cadavres qui sont dans un état de décomposition plus ou moins avancé. Le corps d'un enfant portant un petit drapeau est observé de près. Ce drapeau détermine un changement complet de ton dans le poème. L'enfant, un parmi de nombreux autres, montre que les corps qui couvrent la place appartiennent à un seul peuple. Le Je qui découvre avec effroi le symbole de nationalité, pose sa fourche afin d'enlever le drapeau. Le contact n'est pas sans conséquences. Le symbole de la nation est porteur d'une force contagieuse qui menace directement l'homme à la fourche. Il ne reste plus rien de la légèreté initiale. Le Je découvre qu'il se trouve au milieu du mal et n'apparaît plus dans la dernière strophe. Le poème se termine par un cri d'angoisse. Le 
nationalisme est décrit, dans les quatre derniers vers, comme un mal contagieux auquel on ne peut échapper.

L'image d'une place couverte de cadavres d'une certaine nationalité évoque immanquablement l'extermination des Juifs par les nationaux-socialistes pendant la deuxième guerre mondiale. Lucebert aborde dans «vaalt» l'histoire la plus récente. Il le fait en des termes empruntés à la Kabbale et crée ainsi de la distance par rapport au sujet historiquement si proche. C'est probablement la cause essentielle pour laquelle le poème est resté si impénétrable, alors qu'il possède en même temps une si douloureuse et pénétrante acuité.

«Vaalt» affirme que le mal peut être défini par l'idée de nation. Au regard de l'histoire récente, cela implique que le national-socialisme incarne le mal au $\mathrm{XX}^{\mathrm{e}}$ siècle. Inspiré par le nationalisme et l'illusion raciste, le national-socialisme a produit en Allemagne une politique d'extermination dont la population juive de l'Europe a été la victime.

La thèse développée dans «vaalt» a cependant d'autres implications. Que Dieu soit mêlé aux événements qui ont conduit à la place couverte de cadavres, voilà ce que l'utilisation du symbolisme du mal développé par la Kabbale montre clairement. Parmi les excréments git l'enfant avec le petit drapeau. Dieu est tenu pour responsable d'avoir contaminé le monde du mal inhérent à l'idée de nation, ou d'en être l'origine. Dieu qui secrète le mal comme le corps humain produit ses excrétions, a condamné le peuple d'Israël au statut d'excrément de «vaalt» en le définissant comme une nation.

Les poèmes abordés mettent en lumière une remarquable continuité entre les premiers et les derniers poèmes de Lucebert. «Glorie » est dans cette perspective un poème pour lequel la notion d'élection est aussi cruciale que pour «as alles» et «vaalt». La prédestination du Christ à la mort, exprimée par «het vlees is woord geworden» [la chair s'est faite parole], revient avec l'idée que le Christ est habitué à mourir. Lucebert a créé avec «oh dolorosa», un nouveau poème dans la lignée de «vaalt». Le nationalisme qui frappe la deuxième moitié $\mathrm{du} \mathrm{XX}^{\mathrm{e}}$ siècle est décrit en des termes proches de ceux des premiers poèmes. «Oh dolorosa» confirme, par le lien entre le Christ et la question du langage, le rapport entre «vaalt» et «as alles».

61 Le Christ, parole faite chair, constitue effectivement un exemple pour Lucebert. Il interprète la vie et la mort du Christ comme un fondement pour tenter de s'opposer à la parole de Dieu. Le Christ est une des raisons pour lesquelles le poète écrit. En tant que parole par Dieu prononcée, le Christ le pousse à parler «nauwgezet en wanhopig» [avec exactitude et désespoir] contre la parole de Dieu.

\section{BIBLIOGRAPHIE}

FEIJTER, Anja de : «apocrief / de analphabetische naam». Het historisch debuut van Lucebert in het licht van de intertekst van Joodse mystiek en Hölderlin, De Bezige Bij, Amsterdam, 1994.

LUCEBERT : apocrief / de analphabetische naam, De Bezige Bij, Amsterdam 1952 ${ }^{1}$... $1994^{11}$. verzamelde gedichten, De Bezige Bij, Amsterdam, 1974. 
van de roerloze woelgeest, De Bezige Bij, Amsterdam, 1993.

Ernten im Irrgarten: Neue Gedichte und Zeichnungen, Autorisierte Übertragung aus dem Niederländischen, Auswahl und Herausgabe von Rosemarie Still. Mit einem Guten Morgen Lucebert von Christoph Meckel, Kleinheinrich, Münster, 1990.

Der gestrenge luftikus. Gedichte und Arbeiten auf Papier, Auswahl und autorisierte Übertragung aus dem Niederländischen von Rosemarie Still, Kleinheinrich, Münster, 1994.

34 Pleins Jours en la Compagnie de Lucebert : Promptuaire à travers 220 dessins, Éditions Voix Richard Meier / Elizabeth Krief, Montigny-lès-Metz, 1995.

SCHEER, Lieve : «Lucebert: Miro. Een creatieve interpretatie», Dietsche Warande \& Belfort, $121^{\mathrm{e}}$ année (1976), nº 4. p. 272-280.

SCHOLEM, Gershom : Major Trends in Jewish Mysticism, Schocken Books, New York, $1961^{4}\left[1941^{1}\right.$, $\left.1946^{2}, 1954^{3}\right]$.

TISHBY, Isaiah : The Wisdom of the Zohar. An Anthology of Texts. Systematically arranged and rendered into Hebrew by Fischel Lachower and Isaiah Tishby. With extensive introductions and explanations by Isaiah Tishby. English translation by David Goldstein. 3 volumes, [traduction de Mishnat ha-Zohar, 2 vol., Jérusalem, 1949-1961] The Littman Library of Jewish Civilization, Oxford University Press, New York, 1989.

\section{RÉSUMÉS}

Lucebert (1924-1994) est la figure la plus importante de ce qu'il est convenu d'appeler le Mouvement de Cinquante et qui a profondément transformé la poésie néerlandaise de l'aprèsguerre. Cet article traite de quelques poèmes christiques.

Dans ses premières œuvres où apparaît le Christ comme dans les plus récentes, Lucebert montre clairement que derrière son attaque contre le langage se cache une attaque contre Dieu. La vie et la mort du Christ sont interprétées par le poète comme autant de raisons pour mener un combat pour la parole, de même qu'il trouve dans les réalités historiques des années cinquante les fondements de la nécessité de créer une langue nouvelle. En s'appuyant sur l'intertexte de la mystique juive et de l'œuvre de Friedrich Hölderlin, il conçoit le projet d'une langue nouvelle qu'il concrétise dans de analphabetische naam [le nom analphabétique].

Lucebert (1924-1994) is de belangrijkste vertegenwoordiger van de zogeheten Beweging van Vijftig, die de naoorlogse Nederlandse poëzie ingrijpend veranderd heeft. Dit artikel handelt over een aantal Christusgedichten van Lucebert. In zowel zijn vroege als zijn late gedichten over Christus maakt Lucebert duidelijk dat achter zijn aanval op de taal een aanval op God schuilt. De dichter interpreteert leven en sterven van Christus als reden voor een gevecht om de taal, zoals hij ook de historische werkelijkheid van circa 1950 begrijpt als grond voor de noodzaak om een nieuwe taal te scheppen. Gebruik makend van de intertekst van de Joodse mystiek en van het werk van Friedrich Hölderlin ontwerpt hij zijn programma voor een nieuwe taal, dat hij in de schepping van de analphabetische naam heeft geconcretiseerd.

\section{AUTEURS}

\section{ANJA DE FEIJTER}

Université de Nimègue 